EESTI NSV TEADUSTE AKADEEMIA TOIMETISED. XIV KÖIDE

FOUSIKA-MATEMAATIKA- JA TEHNIKATEADUSTE SEERIA. 1965, NR. 1

ИЗВЕСТИЯ АКАДЕМИИ НАУК ЭСТОНСКОИ ССР. ТОМ ХІV СЕРИЯ ФИЗИКО-МАТЕМАТИЧЕСКИХ И ТЕХНИЧЕСҚИХ НАУК. 1965, N2 1

\title{
Э. ЛИППМАА
}

\section{О ПРИМЕНЕНИИ ДВОЙНОГО РЕЗОНАНСА ПРИ ИССЛЕДОВАНИИ СПЕКТРОВ ЯДЕРНОГО МАГНИТНОГО РЕЗОНАНСА}

Эффекты, вызываемые в ядерной спин-системе возмущающими высокочастотными магнитными полями, могут быть разделены на три группы $[1,8]$ :

1. Возмущение спин-системы под влиянием дополнительного высокочастотного поля $\mathrm{H}_{2}$ с изменением квантовых состояний, появлением новых уровней энергии $\left[{ }^{2},{ }^{3}\right]$ и новых, ранее запрещенных переходов $[4,5,9]$.

2. Отклонение населенностей уровней, непосредственно не возмущаемых полем $\mathrm{H}_{2}$, от равновесного значения и вызываемое этим изменение интенсивностей отдельных спектральных линий (ядерный эффект Оверхаузера) [6,7,8].

3. Влияние на времена жизни отдельных квантовых состояний (релаксационные эффекты).

В случае наблюдения спектра при помощи слабого, далекого от насыщения высокочастотного поля $H_{1}$ и возмущения отдельных линий или мультиплетов высокочастотным сигналом $\mathrm{H}_{2}$ по мере увеличения напряженности поля $\mathrm{H}_{2}$ наблюдаются следующие явления:

линг) [5].

1. $2 \pi|J| \gg|\gamma| H_{2}>\frac{1}{T_{2}^{*}}-$ образование субмультиплетов

(тик-

2. $2 \pi|J| \approx|\gamma| H_{2}$ - усложнение спектра за счет появления новых линий [2].

3. $2 \pi|J| \ll|\gamma| H_{2}$ - максимальное подавление спин-спинового взаимодействия (коллапс) $[10,11]$.

Необходимым условием проявления ядерного эффекта Оверхаузера при отсутствии общих уровней энергии является различие продольных времен релаксации отдельных переходов в спин-системе. В благоприятных условиях ядерный эффект Оверхаузера может наблюдаться $\left[{ }^{7-9,38}\right]$ уже при низком значении напряженности поля $\mathrm{H}_{2}$, когда

4. $\gamma^{2} H_{2}^{2} T_{1} T_{2}^{*} \approx 1$.

Каждый из перечисленных четырех методов открывает новые возможности для интерпретации сложных спектров ЯМР.

Образование субмультиплетов из спектральных линий, имеющих общие уровни энергии с линией, возмущаемой полем $H_{2}$ (режим 1), и ядерный эффект Оверхаузера применяются для определения взаимного расположения уровней энергии спин-системы $[5,7]$. Эти методы, а также методы, основанные на применении промежуточной силы поля $\mathrm{H}_{2}$ (режим 2), используются для точного определения химических сдвигов $\left[{ }^{12}\right]$, для нахождения скрытых линий в сложных спектрах $\left[{ }^{13,14}\right]$ и для определения знаков констант косвенной спин-спиновой связи $[15-18,30,31]$. 
Режим 3 обеспечивает максимальное подавление спин-спинового взаимодействия и используется для упрощения спектра $[4,10]$, но ввиду существования остаточного расщепления [ $\left.{ }^{6,9}\right]$ требует для успешного применения дополнительной частотной модуляции поля $\mathrm{H}_{2}$ [19]. Иногда этот метод используется как дополнительный упрощающий прием в. тройном резонансе $\left[{ }^{20,21}\right]$.

\section{Техника эксперимента}

Каждый из перечисленных вариантов двойного резонанса требует различной напряженности высокочастотного поля (нужный диапазон превышает 60 дб) и может быть осуществлен как разверткой магнитного поля, так и разверткой $\omega_{1}$ («развертка частоты») или $\omega_{2}$ («Индор»$\left.\left[{ }^{22}\right]\right)$. Легче всего реализуется первый метод.

Вторая частота получается за счет амплитудной модуляции магнитного поля $\left[{ }^{23,24}\right]$ или частоты $\left[{ }^{13}\right]$. Иногда применяется модуляция с подавлением несущей $[25,26]$, однополосная модуляция $\left[{ }^{27}\right]$, частотная модуляция $\left[{ }^{20}\right]$ и синтез частоты $\left[{ }^{10,12,26}\right.$. Развертка магнитного поля не приводит, однако, к достаточно ясным и однозначным изменениям в спектре. Магнитное поле может быть стабилизировано лишь суперстабилизатором.

Оба метода частотной развертки требуют обязательного применения сгин-стабилизатора. Обычно применяется схема Примаса $[5,28]$ с тремя $[5,7,9]$ или двумя частотами модуляции $[18,29,30]$. Соответственно, модуляционными боковыми полосами являются либо $\mathrm{H}_{1}$ и $\mathrm{H}_{2}$, либо только $H_{1}$. Модуляционные методы получения стабилизированного поля $\mathrm{H}_{2}$ трудно применимы, если для подавления спин-спинового взаимодействия [19], для изучения ядерного эффекта Оверхаузера [ $\left.{ }^{33}\right]$ или релаксационных процессов [34] требуется дополнительная импульсная или частотная модуляция поля $H_{2}$. Аналогичные трудности появляются и при исследовании двойного резонанса протонов с другими ядрами. В этих случаях, а также в протон-протонном двойном резонансе для получения $H_{1}$ и $H_{2}$ все больше применяют синтез нужных частот [14,35-38]. Практически во всех случаях стабилизация осуществляется стабилизатором Примаса $\left[{ }^{28}\right]$ или Бейкера [ $\left.{ }^{39}\right]$ с недостаточным быстродействием [ $\left.{ }^{41}\right]$. Предложенный нами [35] принцип управления системой синтеза частот $\omega_{1}$ (поле $H_{1}$ ) и $\omega_{2}$ (поле $H_{2}$ ) сигналом от спин-генератора имеет в этом отношении преимущества.

\section{Спектрометр для исследования двойного магнитного резонанса протонов на частоте 40 мәц}

Спектрометр (см. рисунок) работает с двумя датчиками и состокт из канала стабилизации с фазово-синхронизированным спин-генератором [ $\left.{ }^{40}\right]$ $(1,2,3,4,5)$ и измерительного канала с двумя блоками синтеза частоты для получения измерительной частоты $\omega_{1}$ (поле $H_{1}$ ) и возмущающей частоты $\omega_{2}$ (поле $H_{2}$ ). Спектрометр может работать с разверткой измерительной частоты $\omega_{1}$, возмущающей частоты $\omega_{2}$ или с одновременной разверткой $\omega_{1}$ и $\omega_{2}$ (что соответствует развертке магнитного поля). Низкочастотный генератор с линейной разверткой в диапазоне от 4,3 до 5,7 кәи подключается, соответственно, к блокам синтеза частоты 9, 10 или 1. Развертка не влияет на амплитуды высокочастотных полей $H_{1}$ и $H_{2}$. Стабильность прибора определяется термическим дрейфом магнита IM-300 фирмы «JEOL» (Япония) и составляет около 0,1 гџ за время снятия одного спектра. Спектрометр используется для иссле- 
Блок-схема спектрометра:

1 - управляемый осциллятор спи:генератора; 2 - спин-генератор $\left[{ }^{40}\right]$; 3 - низкочастотный генератор; 4 скрещенные катушки датчика спингенератора; 5 - модуляционные катушки спин-генератора; 6 - скрещенные катушки измерительной головки; 7 - модуляционные катушки; 8. - электромагнит IM-300 („JEOL”. Япония); 9 - блок синтеза частоты для получення $\omega_{2} ; 10$ - блок синтеза частоты для получения $\omega_{1} ; 11$ низкочастотный генератор; 12 управляемый низкочастотный генератор; 13 - генератор напряжения развертки; 14 - усилитель высокой частоты; 15 - высокочастотный фазовый детектор; 16 - низкочастотный фазовый детектор; 17 - генератор модуляцнонной частоты; 18 самописец; 19 - осциллоскоп; 20 импульсный модулятор.

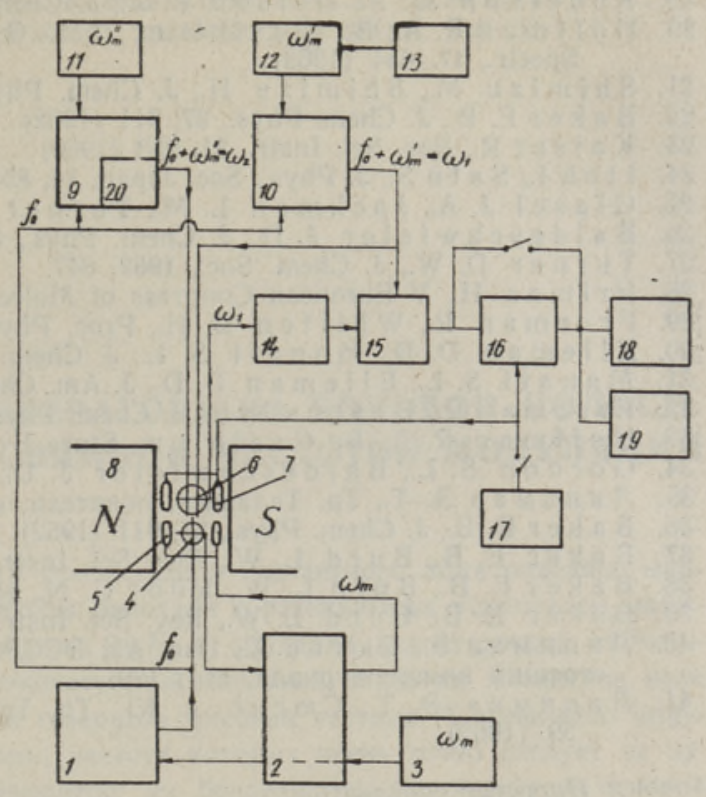

дования образования субмультиплетов (тиклинг) и для подавления спин-спинового взаимодействия (коллапс). Результаты этих исследований будут опубликованы отдельно.

\section{Выводы}

1. Дается обзор методов двойного ядерного магнитного резонанса и применяемых спектрометров.

2. Описан универсальный спектрометр, позволяющий использовать все изложенные варианты двойного протон-протонного ядерного магнитного резонанса.

\section{Л ИТЕ РАТ У Р А}

1. B a 1 deschwiele r J. D., J. Chem. Phys., 40, 459 (1964).

2. B a.lde s chw i el e r J. D., J. Chem. Phys., 34, 718 (1961).

3. Nageswara Rao B. D., Baldeschwieler J. D., J. Chem. Phys., 37, 2473 (1962).

4. B $100 \mathrm{~m} \mathrm{A.} \mathrm{L.,} \mathrm{S} \mathrm{h} \mathrm{o} \mathrm{oler} \mathrm{y} \mathrm{J.} \mathrm{N.,} \mathrm{Phys.} \mathrm{Rev.,} \mathrm{97,} 1261$ (1955).

5. Freem a n R., Anders on W. A., J. Chem. Phys., 37, 2053 (1962).

6. B lo ch F., Phys. Rev., 102, 104 (1956).

7. K a is e r R., J. Chem. Phys., 39, 2435 (1963).

8. Kuhlmann K., B ald es chwieler J. D., J. Amer. Chem. Soc., 85, 1010 (1963).

9. An derson W. A., Freem a n R., J. Chem. Phys., 37, 85 (1962).

10. A nderson W. A., Phys. Rev., 102, 151 (1956).

11. А 6 р а г а м А., Ядерный магнетизм, Изд. иностр. лит., М., 1963.

12. B aldeschwi e ler J. D., R a n d a 11 E. W., Chem. Rev., 63, 81 (1963).

13. Elvi d g e J. A., J a c k m a n L. M., J. Chem. Soc., 1961, 859.

14. Freem a n R., A n der s on W. A., J. Chem. Phys., 39, 806 (1963).

15. M a her J. P., Ev a n s D. F., Proc. Chem. Soc., 1961, 208.

16. Freem a n R., Wh if e n D. H., Mol. Phys., 4, 321 (1961).

17. Freem a n R., Mol. Phys., 4, 385 (1961).

18. Freem a n R., Mol. Phys., 5, 499 (1962). 
19. Anders on W. A., Nels on F. A., J. Chem. Phys., 39, 183 (1963).

20. Hoff man R. A., Bo Gestblom, Salo Gronowitz, Sture Forsen, J. Mol. Spectr., 11, 454 (1963).

21. Shimizu M., Shimizu H., J. Chem. Phys., 41, 2329 (1964).

22. B a ker E. B., J. Chem. Phys., 37, 911 (1962).

23. K a i s e r R., Rev. Sci. Instr., 31, 963 (1960).

24. I t o h I., S a to S., J. Phys., Soc. Japan, 14, 851 (1959).

25. Glase1 J. A., Jackman L. M., Turnet D. W., J. Chem. Soc., 1961, 426.

26. B a 1 d e s chwiele r J. D., J. Chem. Phys., 36, 152 (1962).

27. Turner D. W., J. Chem. Soc., 1962, 847.

28. Prim a S H., V European Congress of Molec. Spectr., Amsterdam, 1961.

29. Freem an R., Whiffen D. H., Proc. Phys. Soc., 79, 794 (1962).

30. E 11 e m a n D. D., M a n a t t S. L., J. Chem. Phys., 36, 2346 (1962).

31. M a n a tt S. L., E11 e m a n D. D., J. Am. Chem. Soc., 84, 1579 (1962).

32. Free m a n R., B h a c c a N. S., J. Chem. Phys., 38, 1088 (1963).

33. H of $\mathrm{fm}$ a n R. A., Bo Gest b 1 o m, Sture For se n, J. Chem. Phys., 39, 486 (1963).

34. Gord on S. L., B a 1 deschwieler J. D., J. Chem. Phys., 41, 571 (1964).

35. Л и п п м а Э. Т., Тр. Таллинск. политехн. ин-та, Сер. А 195, 65 (1962).

36. B a k e r E. B., J. Chem. Phys., 37, 911 (1962).

37. B a k e r E. B., B u r d L. W., Rev. Sci. Instr., 34, 238 (1963).

38. B a ker E. B., B urd L. W., Root G. N., Rev. Sci. Instr., 34, 243 (1963),

39. B a ke r E. B., B u r d L. W., Rev. Sci. Instr., 28, 313 (1957).

40. Л и п п м а Э., С юг и с А., Изв. АН ЭССР. Сер. физ.-мат. и техн. наук. См. настоящий номер журнала, стр. 129.

41. Л и ппм а а Э. Т., С юги с А. Ю., Тр. Таллинск. политехн. ин-та, Сер. А 195, 59 (1962).

Институт кибернетики

Академии наук Эстонской ССР
Поступила в редакцию

$6 / \mathrm{I} 1965$

\section{E. LIPPMAA}

\section{TOPELTRESONANTSI KASUTAMISEST TUUMARESONANTSSPEKTRITE ANALOUSIL}

Antakse ülevaade topeltresonantsi efektidest, meetodeist ja võimalustest spektrite analüüsi lihtsustamise puhul ning kasutatavast aparatuurist.

\section{E. LIPPMAA}

\section{USE OF NUCLEAR MAGNETIC DOUBLE RESONANCE IN THE ANALYSIS OF HIGH-RESOLUTION NMR SPECTRA}

A universal proton-proton nuclear double resonance spectrometer must allow all possible techniques (field sweep, frequency sweep, INDOR) to be used with a broad $(60 \mathrm{db})$ range of perturbing and measuring field strengths, to be spin-stabilized and as free from noise and spurious signals as possible. The amplitudes of the perturbing field $\mathrm{H}_{2}$ and measuring field $\mathrm{H}_{1}$ must be independent of their frequencies - a near impossibility with modulation methods. In some experiments $\mathrm{H}_{2}$ must be available for further pulse or frequency modulation. For these reasons a $40 \mathrm{Mc}$ spectrometer, stabilized by spin generator and using frequency synthesis to obtain $H_{1}$ and $H_{2}$ has been built. Until now the apparatus has been used for tickling and spin decoupling experiments with good results. The drift is about $0.1 \mathrm{cps} / \mathrm{min}$, resolution $1.2 \cdot 10^{-8}$. 